\title{
ROBUST, FFT-BASED, SINGLE CARRIER, MULTIUSER, SDPSK/DPSK BLOCK DEMODULATION IN THE PRESENCE OF SYMBOL SYNCHRONIZATION JITTER
}

\author{
AHMAD A. MASOUD*
}

\begin{abstract}
In this paper a robust, single carrier, multi-user, DSP-based implementation of an OFDM, differential phase shift keying (DPSK) block demodulator is suggested. It is shown that a direct, DSP-based implementation of the above system using a single FFT symbol is highly susceptible to artifacts induced by symbol timing errors (symbol synchronization jitter, SSJ). A dual symbol, FFT-based implementation is suggested to alleviate the effect of timing error on the demodulation process, even eliminate some of the SSJ-induced artifacts. The countermeasures used in the implementation do not assume any statistical model of SSJ nor are they dependant on a symbol synchronization scheme. Also a dual version of the demodulator is derived for an important, but less known, modulation technique called symmetric DPSK (SDPSK).
\end{abstract}

Key words: SDPSK, DPSK, FFT, DSP, block demodulation

\section{Nomenclature}

DSP: $\quad$ Digital signal processing

PSD: $\quad$ Power spectral density

OFDM: Orthogonal frequency division multiplexing

SSJ: Symbol synchronization jitter

FFT: $\quad$ Fast Fourier transform

DFT: Discrete Fourier transform

DPSK: Differential phase shift keying

SDPSK: $\quad$ Symmetric DPSK

VLSI: Very large scale integration

AWGN: Additive white Gaussian noise

BAWGN: Bandlimited AWGN

ICI: Inter channel interference

DPN: Differential phase noise

PE: Probability of error

$E_{b}$ : $\quad$ Symbol energy per bit duration

$N_{o}$ : Power spectral density of AWGN

$N$ : Number of samples per bit duration

$L: \quad$ Number of users

* Electrical Engineering Department, KFUPM, P.O. Box 287, Dhahran 31261, Saudi Arabia, E-mail: masoud@kfupm.edu.sa, Tel: 03-860-3740 Fax: 03-860-3535. 

T: Symbol duration
$\Delta T: \quad$ Sampling period
$f_{s}: \quad$ Sampling frequency
c: $\quad$ Carrier frequency

1. Introduction. Differential phase encoding is highly desirable in situations where carrier synchronization cannot be reliably performed. Differential phase shift keying (DPSK) [1,2], is a popular form of differential phase modulation that encodes the alphabets of a link by rotating the phase of the carrier by $\pi$ if the alphabet 0 is transmitted, and by 0 (i.e. leaving the phase unchanged) if 1 is transmitted. A variation on DPSK, called symmetric DPSK (SDPSK), has received considerable attention in the literatrue [3-8]. SDPSK encodes the alphabets by rotating the phase of the carrier by $-\pi / 2$ if 0 is transmitted, and by $+\pi / 2$ if 1 is transmitted. In an ideal situation the performance of SDPSK was found to be similar to that of DPSK. However, when the operating conditions deviate a little from the ideal, the performance of SDPSK becomes superior to that of DPSK. Winters [6] found that when intersymbol interference is present, SDPSK maintains equal error rates for all data bits. This is in contrast to DPSK where the error rate for 1's can be several time higher than that for 0's. This disparity in probabilities of error (PE's) is highly undesirable, and is disruptive to the operation of error correcting codes. Also, the symmetric nature of phase transition in an SDPSK signal has been found to yield a relatively constant envelope after filtering. This results in SDPSK being less likely to suffer distortion due to hard limiting than DPSK. This desirable feature of SDPSK was further enhanced by Alexovich et al. [7] through the use of Bandpass-limited SDPSK. Winters [6] also found that SDPSK has a lower bit error rate in the presence of timing error and frequency offset than DPSK. In [8] Yasheng et. al noted that, equipped with anti-multipath measures, SDPSK offers a better performance than DPSK. The advantages that SDPSK has over DPSK, at least as far as the symbol timing issue is concerned, are obvious. DPSK is highly likely to face problems in symbol synchronization when long runs of the same bit are encountered. Long runs of the same bit do happen even if the data stream is whitened. In an SDPSK encoded signal a component having the fundamental frequency of $1 / T$ will always be present in the received signal regardless of the statistical distribution of the data stream. This enables the synchronizing circuitry, at all times, to accurately determine the beginning and end of a symbol.

It is highly desirable to have a DSP-based realization of a demodulator. DSP implementations are highly compact, they consume little power, and they are able to utilize the wealth of DSP algorithms to enhance the performance of a receiver. Of particular interest is the VLSI-friendly, fast Fourier transform (FFT) algorithm 
[9]. FFT naturally lends itself to the block demodulation of differentially, phase encoded signals. In this paper, a robust FFT-based, single carrier, multiuser, block demodulator is suggested for DPSK and SDPSK encoded signals.

Section 2 contains background material about DPSK and SDPSK. In Section 3, an FFT-based, block demodulator is suggested for DPSK and SDPSK signals when perfect timing can be achieved. Section 4 discusses the effect SSJ has on the communication link. In Section 5 countermeasures to combat SSJ are suggested and integrated in the block demodulator. Simulation results and conclusions are placed in Sections 6 and 7 respectively.

2. Background. In the following, optimum DPSK and SDPSK are briefly reviewed for the binary case.

2.1. Optimum DPSK. The block diagram describing a DPSK modulator is shown in Figure-1 below :

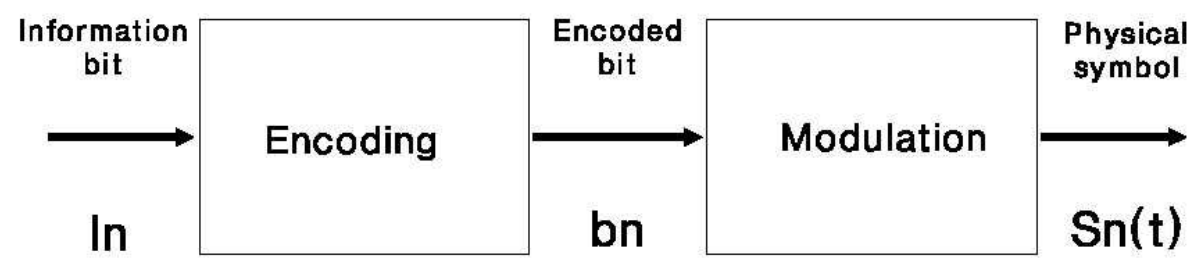

FIG. 1. Block diagram of a DPSK modulator.

Prior to modulation, an information bit $\left(I_{n}\right)$ is encoded. The encoded bit $b_{n}$ is generated using the formula:

$$
b_{n}=b_{n-1} \bigoplus I_{n}
$$

where $\bigoplus$ is the logical exclusive OR operation, and $b_{0}=1$. The symbol to be modulated by the carrier $\left(\cos \left(\omega_{c} t\right)\right)$ and transmitted through the channel $\left(S_{n}(t)\right)$ belongs to the antipodal set of signals $\left\{S_{1}(t), S_{2}(t)\right\}$ where:

$$
S_{1}(t)=A \cdot \cos (\omega t), \& \quad S_{2}(t)=-A \cdot \cos (\omega t), t \in[0, T),
$$

where $A$ is the amplitude of the symbol, and $T$ is its duration. Normally, $\omega$ is set to zero $(\omega=0)$. However, here values besides zero will be used provided that $\omega<<\omega_{c}$. The symbols to be transmitted are selected as follows :

$$
\text { if } b_{n}=1, S_{n}(t)=S_{1}(t), \text { if } b_{n}=0, S_{n}(t)=S_{2}(t) .
$$

It can be easily shown that if $I_{n}=1, S_{n}(t)$ and $S_{n-1}(t)$ are antipodal. If $I_{n}=$ $0, S_{n}(t)$ and $S_{n-1}(t)$ are similar. Let $\hat{S}_{n}(t)$ and $\hat{S}_{n}(i)$ be the n'th continuous-time, 
and discretized symbols respectively, where $i$ is the time index, and the sampling rate $f_{s}=1 / \Delta T, N \times \Delta T=T, i=0, \ldots, N-1$ (Figure 2).

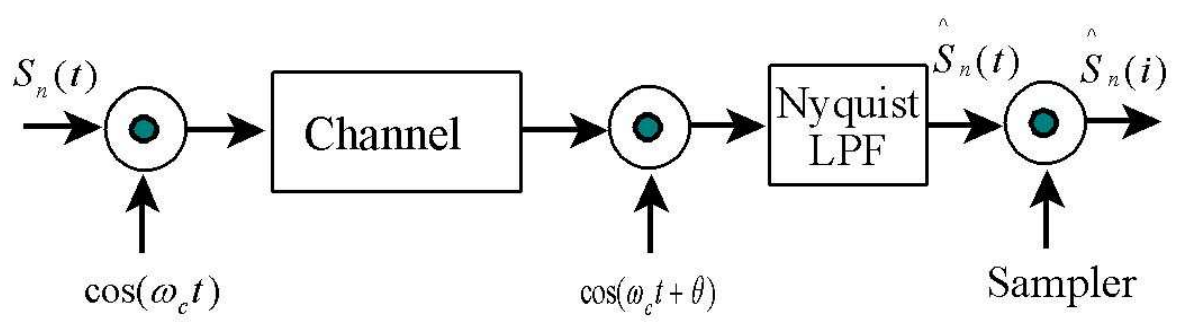

FIG. 2. The transmitted, received and sampled symbols. The channel is assumed to add only white gaussian noise.

Let $\eta_{n}$ be a 2 -D phasor describing the n'th, discrete, received symbol $\left(\eta_{n}=\right.$ $\left.\left[\eta c_{n}, \eta s_{n}\right]^{t}\right)$, where:

$$
\begin{aligned}
\eta c_{n} & =\sum_{i=0}^{N-1} \hat{S}_{n}(i) \cdot \cos \left(\frac{\omega T}{N} i\right), \\
\eta s_{n} & =\sum_{i=0}^{N-1} \hat{S}_{n}(i) \cdot \sin \left(\frac{\omega T}{N} i\right) .
\end{aligned}
$$

To make a decision regarding the n'th received bit, the following decision variable constructed using the inner product of $\eta_{n}$ and $\eta_{n-1}$ is used:

$$
\gamma_{n}=\eta_{n}^{t} \eta_{n-1}=\eta c_{n} \eta c_{n-1}+\eta s_{n} \eta s_{n-1} .
$$

If $\gamma_{n} \geq 0$, then a decision is made that $I_{n}=1$ was transmitted, else a decision is made that $I_{n}=0$ was transmitted.

2.2. SDPSK. SDPSK encodes data by rotating the phasor $\eta_{n}$ counter-clockwise if $I_{n}=1$, and clockwise if $I_{n}=0$, or simply

$$
S_{n}(t)=A \cdot \cos \left(\omega t+\frac{\pi}{2} \cdot a_{n}\right),
$$

where $a_{n}=a_{n-1}-1$, for $I_{n}=0$, and $a_{n}=a_{n-1}+1$, for $I_{n}=1, a_{0}=0$. Similar to DPSK, decoding an SDPSK signal starts by constructing the phasor $\eta_{n}$. Since the direction of rotation of the phasor determines the information bit that was transmitted, the decision variable is constructed using the cross product of $\eta_{n}$ and $\eta_{n-1}$ :

$$
\gamma_{n}=\eta_{n} \times \eta_{n-1}=\eta c_{n} \eta s_{n-1}-\eta s_{n} \eta c_{n-1} .
$$

If $\gamma_{n} \geq 0$, a decision is made that $I_{n}=1$ was transmitted, else a decision is made that $I_{n}=0$ was transmitted. It ought to be mentioned that since a Nyquist lowpass filter is used to band-limit the signal prior to sampling, the samples of the 
band-limited, additive, white, gaussian, noise (BAWGN) are independent. Therefore, the decision criteria (5) and (7) are expected to remain optimum for the discrete case. Simulation results (See section-6) seem to support this argument.

2.3. Frequency Domain Multi-User Encoding. A symbol may be used to encode more than one information bit (Figure 3).

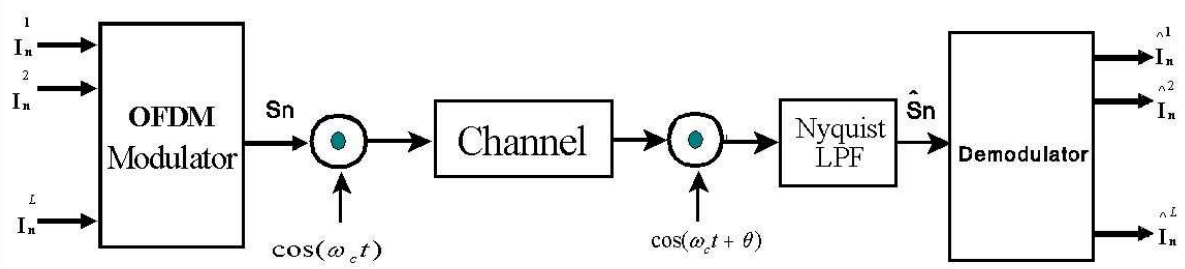

FIG. 3. A symbol encoding $L$ information bits.

OFDM is one way to synthesize a symbol with an informational equivalence of $L$ information bits (from now on, information bits will be referred to as users or channels):

$$
S_{n}(t)=A \sum_{k=0}^{L-1} \cos \left(\frac{2 \pi}{T} k t+\phi_{n}^{k}\right)
$$

where $k$ denotes the channel or user number, and $\phi_{n}^{k}$ is the phase sent by the k'th user during the n'th transmission.

\section{Single FFT, OFDM, Block Demodulators for DPSK and SDPSK}

Encoded Signals. The discrete Fourier transform (DFT) of $\hat{S}_{n}(i)$ is :

$$
F_{n}(k)=\operatorname{DFT}\left(\hat{S}_{n}(i)\right)=\sum_{i=0}^{N-1} \hat{S}_{n}(i) \cdot \cos \left(\frac{2 \pi}{N} k i\right)-j \sum_{i=0}^{N-1} \hat{S}_{n}(i) \cdot \sin \left(\frac{2 \pi}{N} k i\right)
$$

As can be seen the phasor vector in (4), which is needed to construct the decision variable for the k'th channel, is equal to the k'th component of the DFT of the received symbol, i.e.

$$
\eta_{n}^{k}=F_{n}(k), \quad \eta c_{n}^{k}=\operatorname{Re}\left(F_{n}(k)\right), \& \eta s_{n}^{k}=\operatorname{Im}\left(F_{n}(k)\right)
$$

Note that FFT is only a fast implementation of DFT. The n'th bit from the k'th user may be decoded as follows:

a. DPSK (Figure 4a)

$$
\gamma_{n}(k)=\operatorname{Re}\left(F_{n}(k)\right) \operatorname{Re}\left(F_{n-1}(k)\right)+\operatorname{Im}\left(F_{n}(k)\right) \operatorname{Im}\left(F_{n-1}(k)\right)
$$

If $\gamma_{n}(k) \geq 0, I_{n}^{k}=1$. If $\gamma_{n}(k)<0, I_{n}^{k}=0$. 
b. SDPSK (Figure 4b)

$$
\gamma_{n}(k)=\operatorname{Re}\left(F_{n}(k)\right) \operatorname{Im}\left(F_{n-1}(k)\right)-\operatorname{Im}\left(F_{n}(k)\right) \operatorname{Re}\left(F_{n-1}(k)\right) .
$$

If $\gamma_{n}(k) \geq 0, I_{n}^{k}=1$. If $\gamma_{n}(k)<0, I_{n}^{k}=0$.

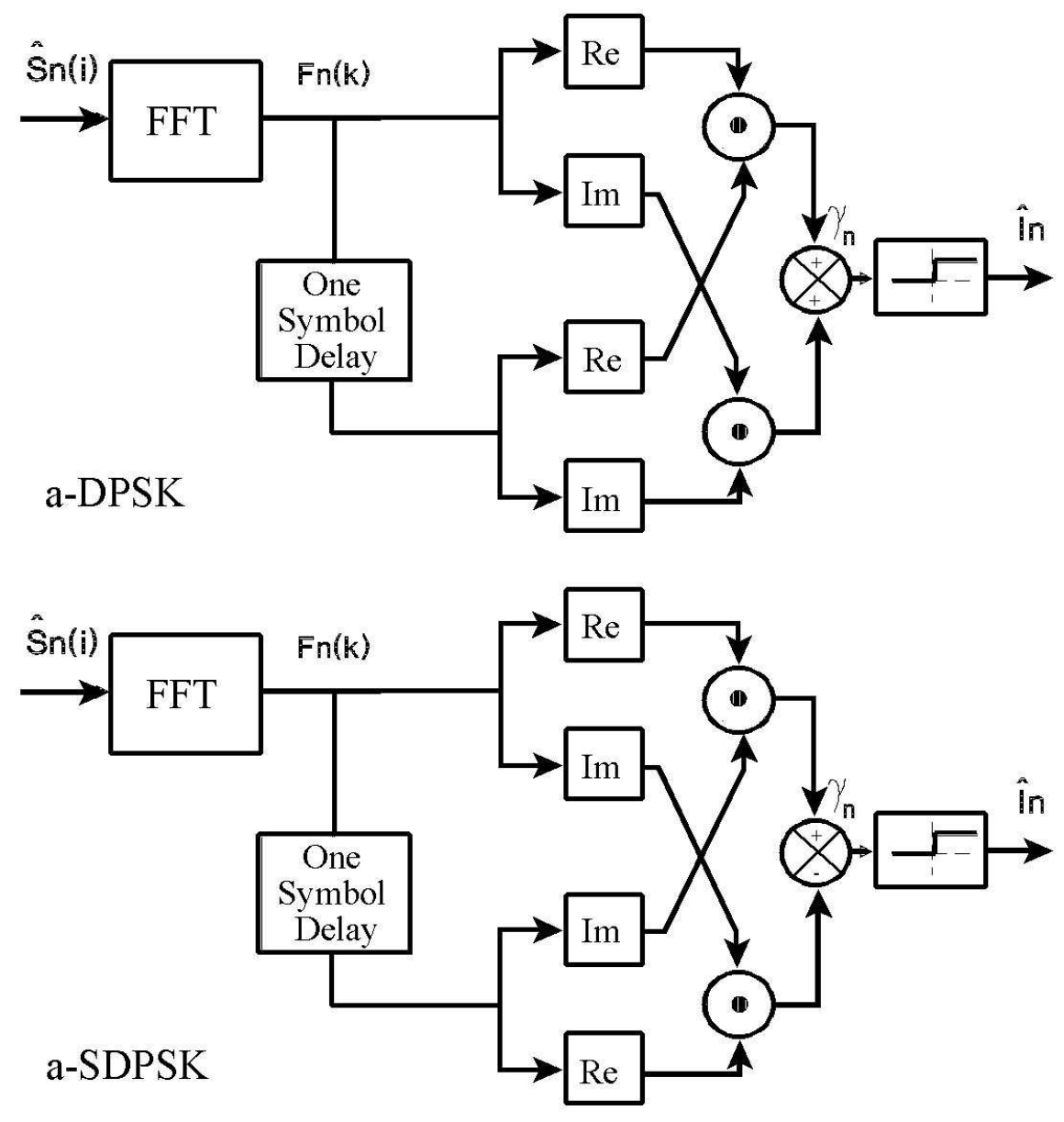

FIG. 4. FFT-based, block demodulator, SSJ-free case.

4. The SSJ Effect on the Communication Link. In the following, two ways in which SSJ [11] can impact on performance are discussed:

4.1. Inter-channel Interference (ICI). SSJ tampers with the orthogonality of the symbols used by the different users. This causes the power from one user to leak to another. Figure 5a shows the power concentration from a single user. Figure $5 \mathrm{~b}$ shows the SSJ-induced power-spill to other users caused by SSJ.

4.2. Differential Phase Noise (DPN). SSJ also causes relative phase disturbance. By disturbing the relative phase between two successive symbols SSJ poses 


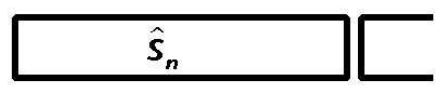

Acquisition buffer

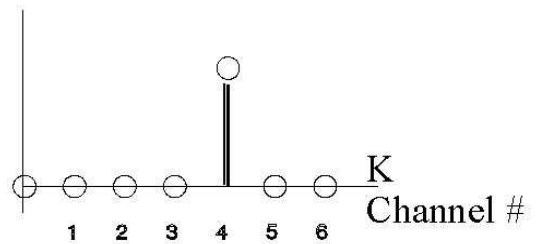

SSJ-Free Case A
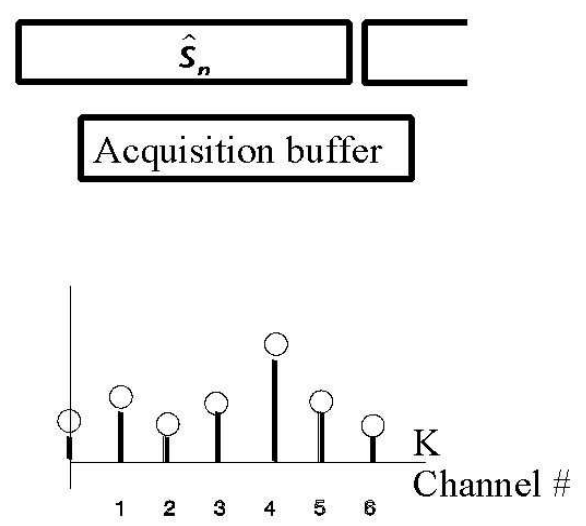

SSJ-Present B

FIG. 5. Effect of SSJ on the power distribution of a channel.

the threat of rendering the received signal unusable for decoding the information contained in its differential phase. This serious artifact arises due to the misalignment between two successively collected signals representing two successive symbols. This is a direct result of the failure of the synchronization circuitry to accurately determine the start and end of the received symbols. Differential phase noise $\left(\Delta \theta_{n}^{k}\right)$ unevenly affects individual channels with high frequency users being more susceptible to this type of noise than low frequency ones. Let us assume that due to inaccurate localization of the beginning and end of a received symbol the n'th symbol begins registering in the collection buffer at time $t=\Delta_{n} \cdot T$ instead of $t=0$ (Figure 6 ). In the same way the acquisition of the $n-1^{\prime}$ th symbol begins at $t=\Delta_{n-1} \cdot T$. Both $\Delta_{n}$ and $\Delta_{n-1}$ are independent random variables uniformly distributed between $(-.5, .5)$. As can be seen from Figure 6, relative to the receiver, a random misalignment of symbol $S_{n}$ with respect to $S_{n-1}$ of the amount $\left(\Delta_{n}-\Delta_{n-1}\right) \cdot T$ has occurred. To compute how much phase disturbance this misalignment causes in each channel, we need first to compute the duration of one cycle in the k'th channel $\left(T_{k}\right): T_{k}=T / k$. Since the duration of one cycle in a channel corresponds to a $2 \pi$ phase shift, the misalignment causes the following differential phase shift in the k'th channel:

$$
\Delta \theta_{n}^{k}=2 \pi \frac{\Delta_{n}-\Delta_{n-1}}{T_{k}} T=2 \pi\left(\Delta_{n}-\Delta_{n-1}\right) k .
$$

As can be seen, differential phase noise intensifies as the frequency of a user in the OFDM transmitted signal becomes higher. Choosing $\Delta_{n}$ and $\Delta_{n-1}$ as uniformlydistributed random variables in order to model SSJ is the worst case scenario that can take place as far as symbol synchronization is considered. Designing an effective block demodulator for this case will likely yield a robust performance. 


\section{SSJ Countermeasures.}

5.1. Removing Differential Phase Noise. The most serious SSJ-induced artifact is the differential phase noise. The severity of this artifact is simulated for two users using two channels obtained by setting the orthogonal frequency index to $k=1$ and $k=7$. The demodulator uses a 16-sample FFT which corresponds to a maximum capacity of 8 users. The DPSK receiver is constructed using the procedure in Section 3. Table 1 shows the PE computed over 200,000 transmissions at medium levels of signal to noise (i.e. $E_{b} / N_{o}$ ) for the SSJ-free case and a small value of SSJ. As can be seen for the SSJ-free case, the PE is the same for both the high and low frequency users. However, the situation dramatically changes when a small amount of SSJ is introduced. While the low frequency user suffers some degradation in performance, the performance of the high frequency user suffers serious degradation rendering the channel unusable for transmitting information. It is worth mentioning that $6.25 \%$ SSJ is less than one sample error in the 16-sample block receiver. This reveals how sensitive the block demodulation process can be to even small errors in timing. It ought to be mentioned that the above results are obtained for the worst case of independent SSJ between adjacent samples. In general, the symbol synchronization circuit with a low pass filter would generate correlated SSJ.
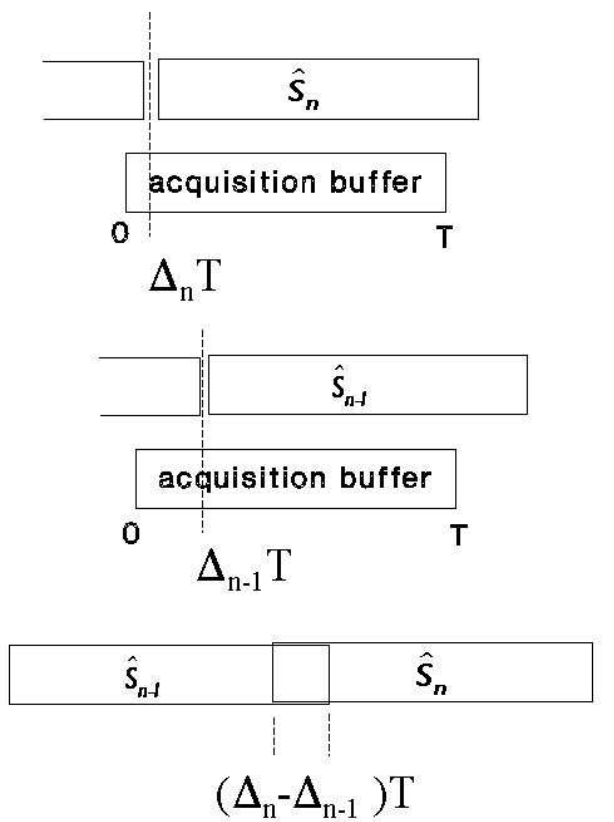

\section{Collection of the n'th symbol}

\author{
Collection of the n-1'th \\ symbol
}

\section{Symbols as they appear to the FFT-based receiver}

FIG. 6. SSJ causing symbol misalignment.

Fortunately, SSJ-induced DPN can be totally eliminated by slightly modifying the FFT-based receiver in Section 3. The idea is simple: instead of restricting the 
TABLE 1

Effect of SSJ on PE, 2-channel, 16 sample, DPSK.

\begin{tabular}{|c|c|c|c|}
\hline Jitter \% of $T$ & $E_{b} / N_{o}$ & $P_{e}$ & $P_{e}$ \\
& & $k=1$ & $k=7$ \\
0.00 & 6.25 & 0.0010 & 0.0010 \\
0.00 & 4.00 & 0.0088 & 0.0085 \\
6.25 & 6.25 & 0.0093 & 0.4990 \\
6.25 & 4.00 & 0.0414 & 0.5070 \\
\hline
\end{tabular}

acquisition buffer to the duration of one symbol only, it is made large enough to accommodate two symbols (Figure 7).

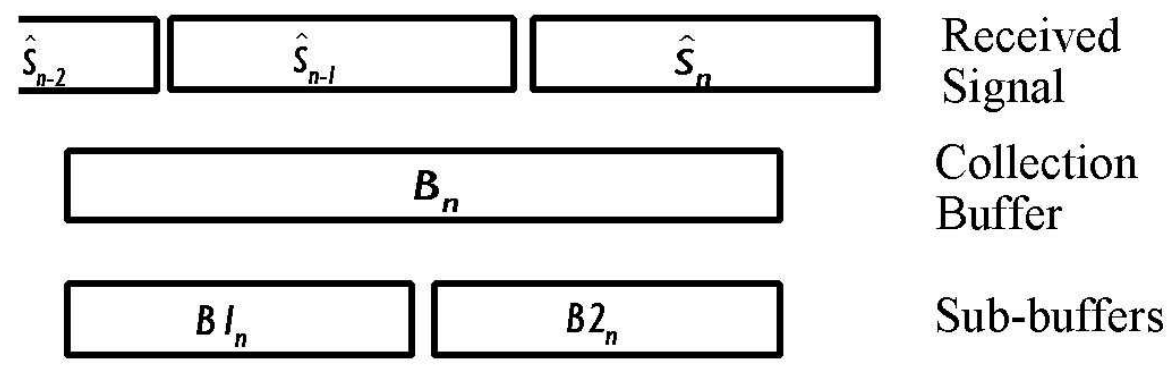

FIG. 7. An approach to eliminate frame misalignment.

The signal in the collection buffer is split into two sub-buffers each having the duration of one symbol. This arrangement makes sure that no misalignment between the $n^{\prime}$ th and $n-1^{\prime}$ th symbol can occur causing DPN. The new procedure for decoding the $n$ 'th symbol is:

1- acquire the signal $B_{n}(i), i=0, \ldots, 2 N-1$;

2- construct the following signals

$$
B 1_{n}(j)=B_{n}(j), B 2_{n}(j)=B_{n}(j+N) .
$$

3- compute:

$$
\begin{aligned}
& F 1_{n}(k)=F F T\left(B 1_{n}(j)\right), \\
& F 2 n(k)=F F T(B 2 n(j)), j=0, . ., N-1 .
\end{aligned}
$$

4- in the case of DPSK (Figure 8a):

$$
\gamma_{n}(k)=\operatorname{Re}\left(F 1_{n}(k)\right) \operatorname{Re}\left(F 2_{n}(k)\right)+\operatorname{Im}\left(F 1_{n}(k)\right) \operatorname{Im}\left(F 2_{n}(k)\right) ;
$$

5- in the case of SDPSK (Figure 8b):

$$
\gamma_{n}(k)=\operatorname{Re}\left(F 2_{n}(k)\right) \operatorname{Im}\left(F 1_{n}(k)\right)-\operatorname{Im}\left(F 2_{n}(k)\right) \operatorname{Re}\left(F 1_{n}(k)\right) .
$$



6- If $\gamma_{n}(k) \geq 0, I_{n}^{k}=1$. If $\gamma_{n}(k)<0, I_{n}^{k}=0$.

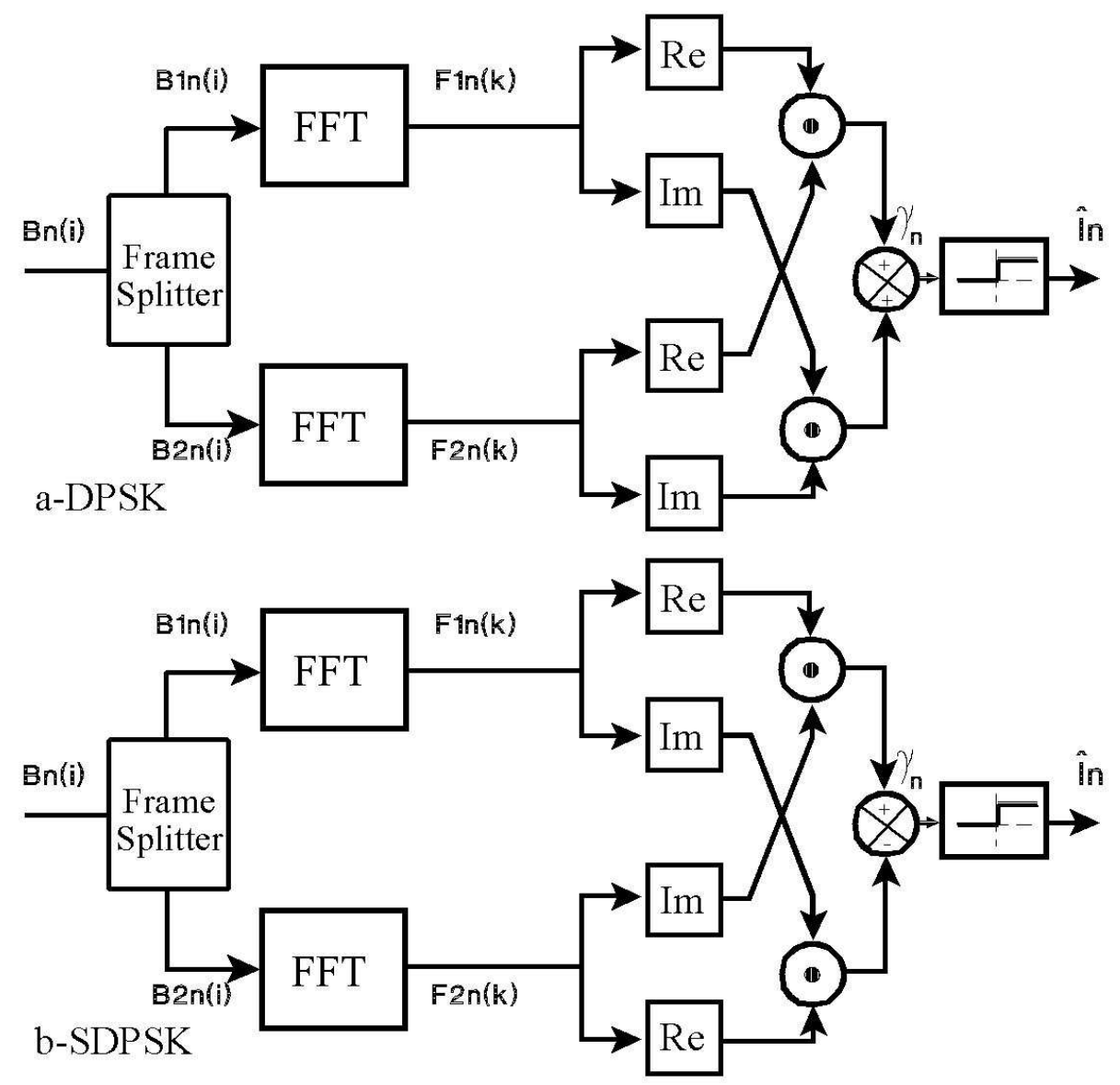

FIG. 8. SSJ-resistant, FFT-based demodulator.

Eliminating SSJ-induced DPN is carried out at the expense of slightly increasing the computational effort that is needed to decode one symbol. In The SSJ-resistant receiver two FFTs are needed to decode one symbol instead of one FFT as in the SSJ-free case. Since both FFTs are executed in parallel, the receiver experiences no slowdown in operation.

5.2. Reducing Inter-channel Interference by Windowing. Reducing the SSJ-induced power leakage may be achieved by reducing the importance of the peripheral samples of the data in the sub-buffers. One way of accomplishing this is by multiplying the data in the sub-buffers by a proper window function [10] prior to performing FFT. This can significantly confine the leakage of power from the users 
and significantly reduce PE. Since the front and tail portions of the symbol buffer are attenuated, a drop in SNR and an increase in PE are expected. Therefore, the choice of the window parameters has to be made dependant on SSJ. Also, windowing tends to concentrate power in the vicinity of a user (main lobe of a window). While interference with distant users is reduced, if one does not carefully select the window function, interference with nearby users could increase. The use of windowing can only result in a tradeoff situation that requires a good judgement call on part of the designer. Nevertheless, it is found that common window functions (e.g., a hamming window) can significantly reduce the effect of ICI on performance.

6. Simulation Results. In this section simulation results of the suggested DPSK and SDPSK receivers are presented. The receivers are required to decode the symbols from four users. A 16-point FFT is used where the users are assigned the pins $1,3,5,7$ respectively. The probability of error for each user is computed for different values of $E_{b} / N_{o}$, and percentages of SSJ by averaging the errors from 500,000 transmissions. While the PE is computed for each user separately, the average PE for the four users is used as the measure of the link's performance. Also, for the discrete case, $E_{b} / N_{o}$ (symbol energy per bit duration divided by the power spectral density (PSD) of the AWGN) may be written as:

$$
\frac{E_{b}}{N_{o}}=\frac{A^{2} N}{4 \sigma_{n}^{2}}
$$

where $A$ is the magnitude of the sinusoidal signal, $N$ is the number of samples per symbol duration $(T)$, and $\sigma_{n}^{2}$ is the variance of the noise samples. For the SSJ-free case, it is found that the PEs of DPSK and SDPSK are the same and are both equal to $\frac{1}{2} \exp \left(-E_{b} / N_{o}\right)$ which is the well-known probability of error for optimum DPSK in the continuous time case.

The receiver is simulated for two types of windows: rectangular and Hamming. It can be seen from figures 9 and 10 that the choice of a window has a pronounced influence on the probability of error. When no windowing is used PE quickly deteriorates with an error in timing. On the other hand the hamming window, tends to produce a PE that slowly increases with timing errors. Unfortunately, this is achieved at the expense of significantly increasing PE for the SSJ-free case. Those observations hold true for both DPSK and SDPSK links. However, as expected, the growth of PE as a function of SSJ seems slower for SDPSK than it is for the DPSK case.

7. Conclusions. A prototype of a block demodulator that may be able to provide backbone capabilities to a high-capacity, high-performance communication link is suggested. The development is carried out with the application of processing and switching on-board a mobile platform in mind. To deal with the stringent requirements of the above situation, a DSP-based, VLSI-friendly, FFT-based approach to 
receiver synthesis is adopted. Countermeasures to combat the performance-degrading artifacts that are induced by SSJ are proposed and integrated in the structure of the receiver.

It ought to be emphasized that the impact of SSJ on the block demodulation process is severe even if SDPSK is used for modulation and SSJ is significantly correlated. The example in table-1 clearly shows that a maximum SSJ of a single sample error is enough to cripple the communication link for high frequency users $\left(P_{e}=1 / 2\right)$. Equation (13) in the paper clearly shows that the intensity of DPN is linearly proportional to the user's digital frequency index $(k)$. In a realistic situation a communication link may be designed to be sampled at 1024 sample per symbol, i.e. the link can ideally carry 512 users. This means that the intensity of differential phase noise experienced by high frequency users is amplified hundreds of times compared to that of the low frequency users. From this one concludes that the structure suggested in this paper is necessary for a reliable multiuser communication link to be established even if a high precision synchronization circuit is jointly used with SDPSK and SSJ is significantly correlated.

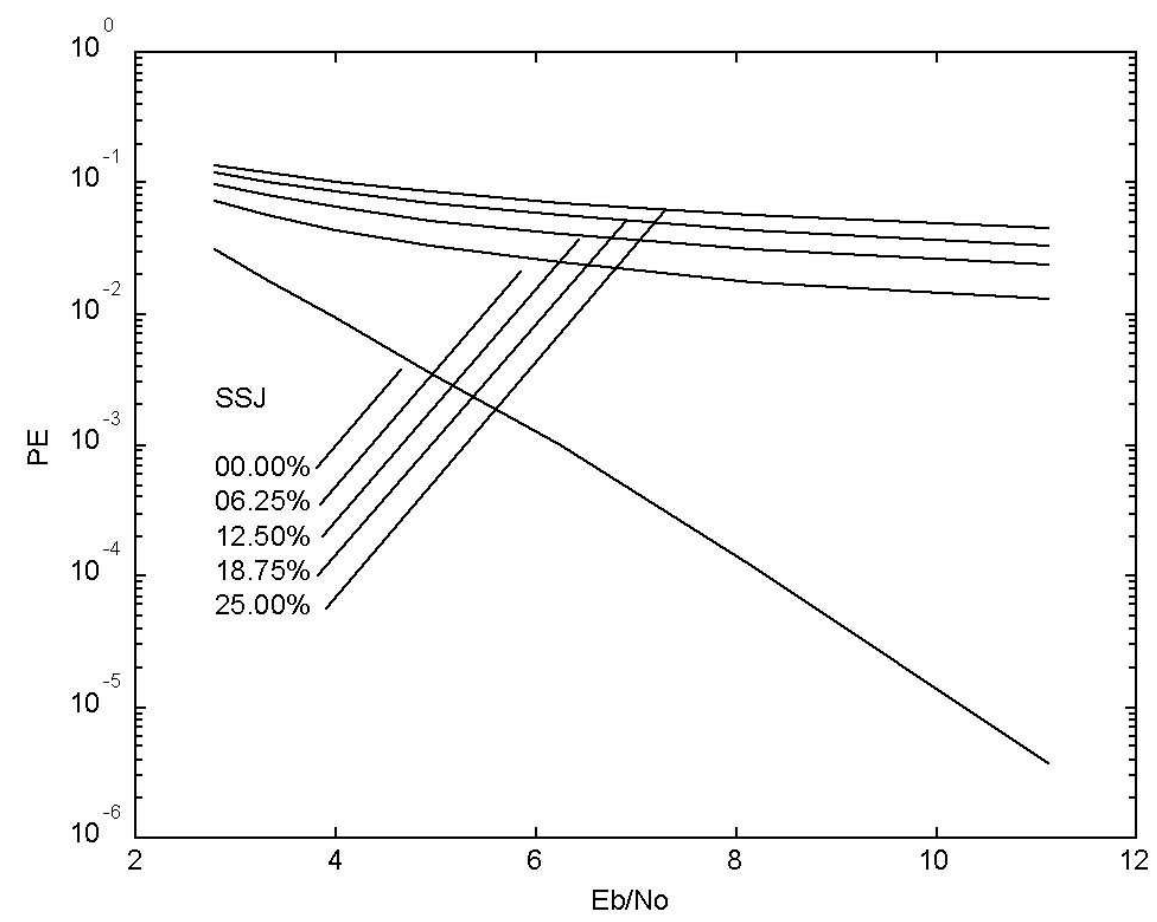

FIG. 9. (a) DPSK-Rectangular window.

Acknowledgment. The author wold like to thank Dr. Aziz Qureshi for his assistance that made it possible to finish this work. The author would also like to 


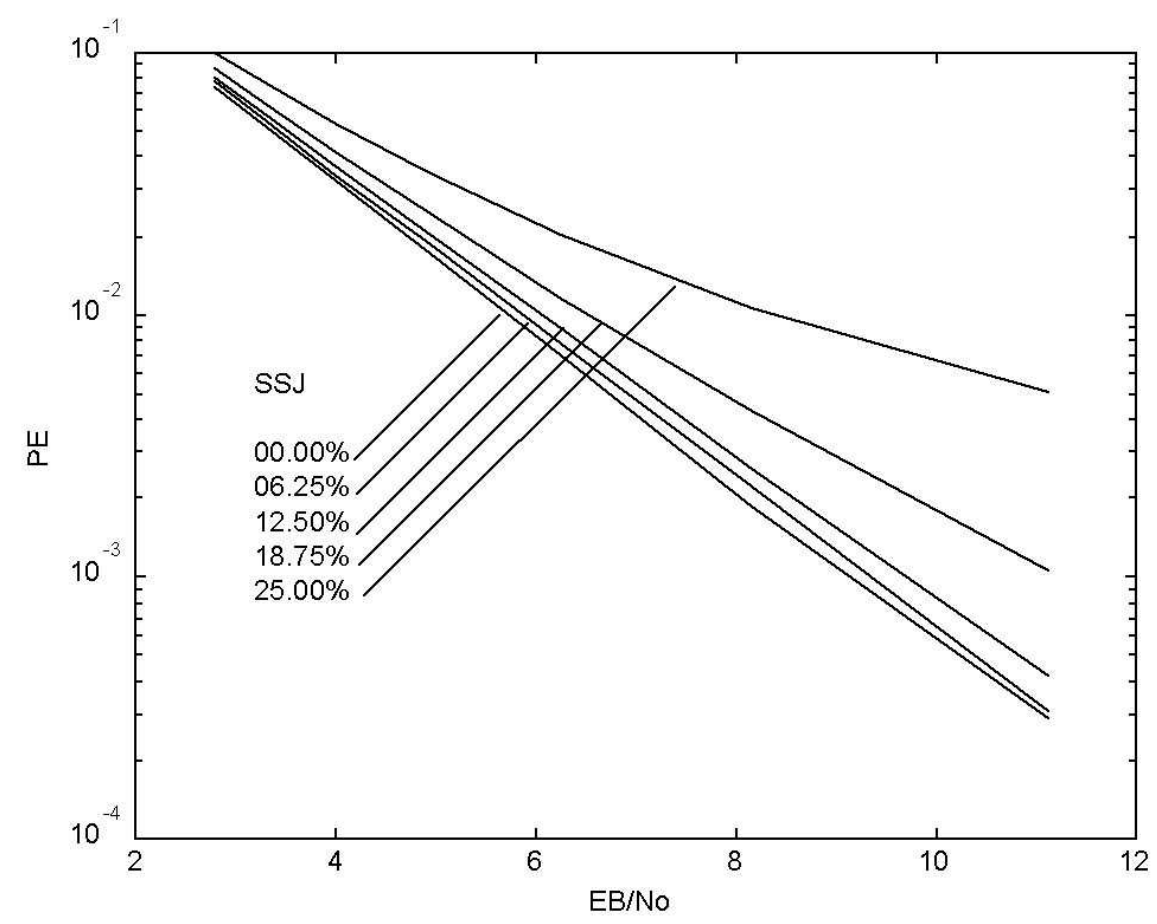

FIG. 10. (b) DPSK-Hamming window.

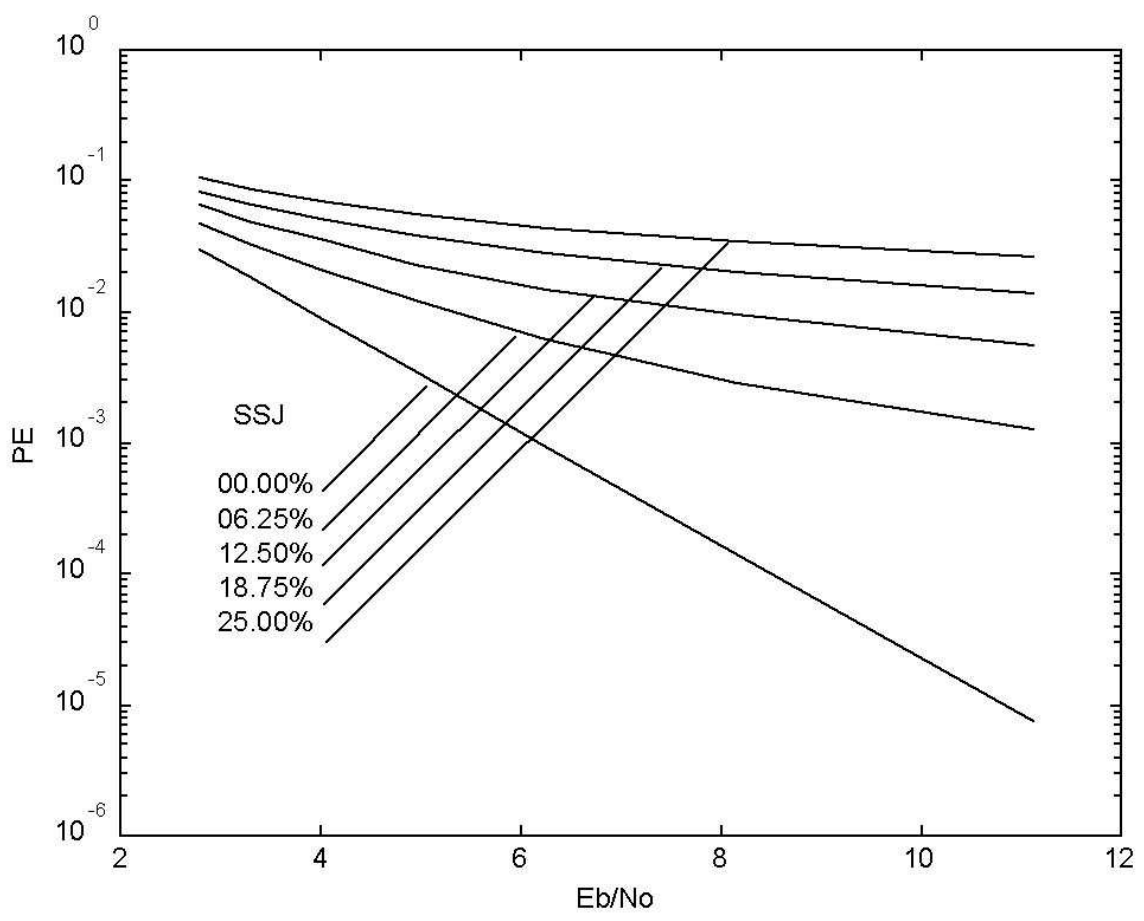

FIG. 11. (a) SDPSK-Rectangular window. 


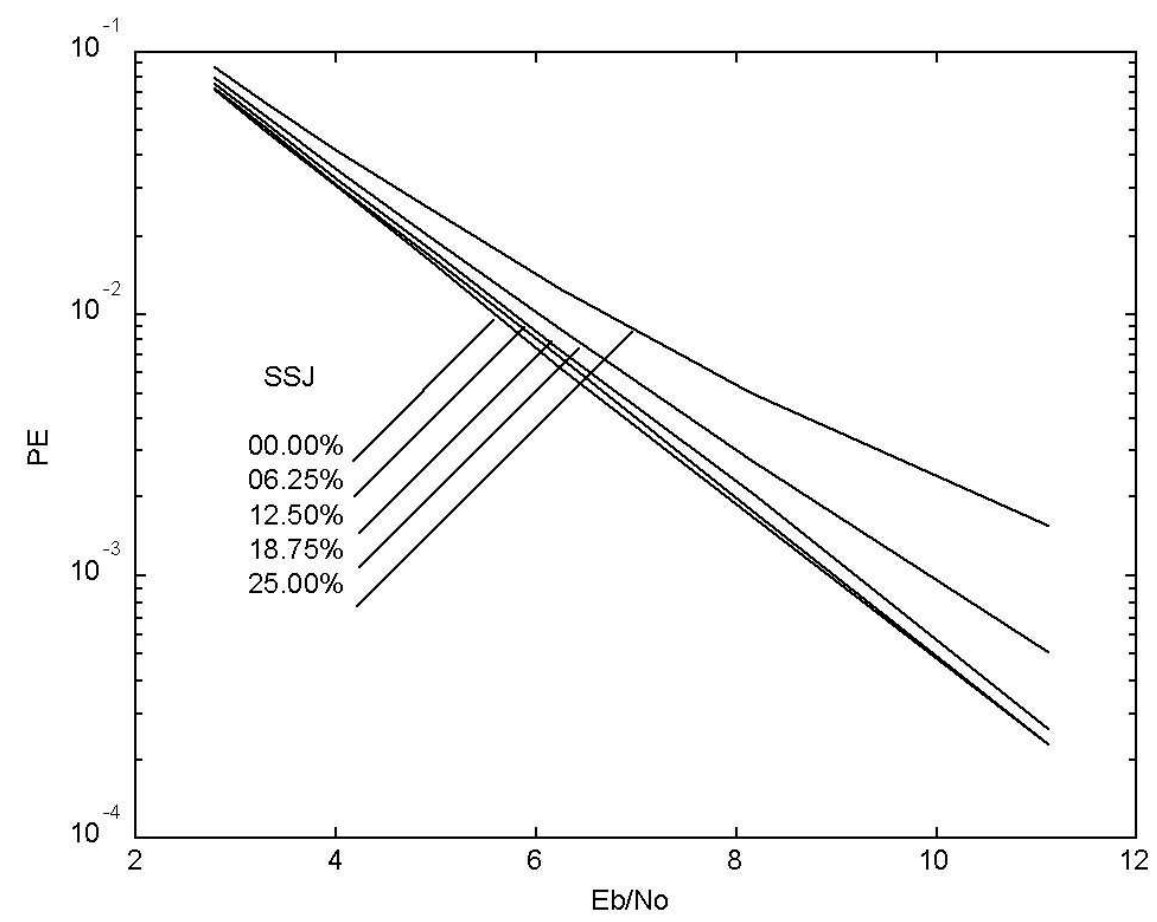

FIG. 12. (b) DPSK-Hamming window.

thank King Fahd University of Petroleum and Minerals for its support. The author would also like thank the anonymous reviewers for their helpful comments.

\section{REFERENCES}

[1] G. Proakis, Digital Communications, Second Edition, McGraw-Hill Book Company, 1989.

[2] R. Ziemer and W. Tranter, Principles of Communications, Systems, Modulation, and Noise, Third Edition, Houghton Mifflin Company, Boston, 1990.

[3] W. HubBard, The Effect of Intersymbol Interference of Error Rate in Binary DifferentiallyCoherent Phase-Shift-Keyed Systems, Bell System Technical Journal, 46, July-August, 1967, pp. 1149-1172.

[4] M. Schwartz, W. Bennett, And S. Stein, Communication Systems \& Techniques, McGrawHill, 1966.

[5] F. Chetnick, Analysis and Tests of Quadrature Advance/Retard Keying Modulation \& Detection, Aeronutronic Ford Corporation (technical memorandum), August 1974.

[6] JACK H. Winters, Differential Detection with Intersymbol Interference and Frequency Uncertainity, IEEE Transactions on Communications, Vol. COM-32, No.1 January 1984, pp. 25-33.

[7] J. R. Alexovich, P. S. Kossin, And T. A. Schonhoff, Bandpass-limited Symmetric DPSK: a bandwidth-efficient modulation for nonlinear channels, Military Communication Conference, 1992, MILCOM, Vol. 3, pp. 827-831.

[8] Q. Yasheng And H. Leib, An Enhanced Anti-multipath Modulation Approach 
(SDPSK/SMPDI), 1996 IEEE International Conference on Communications, ICC'96, Vol. 1, pp. 369-373.

[9] G. Proakis and D. Manolakis, Digital Signal Processing, Principles, Algorithms, and Applications, Second Edition, Macmillan Publishing company, 1992.

[10] F. HARRIS, On the Use of Windows for Harmonic Analysis with the Discrete Fourier Transform, Proceeding of the IEEE, 66:1(1978), pp. 51-83.

[11] P. Trischitta and E. Varma, Jitter in Digital Transmission Systems, Artech House, 1989. 
AHMAD A. MASOUD 